\title{
Antiferromagnetism in the organic conductor bis-tetramethyltetraselenafulvalene hexafluoroarsenate [(TMTSF)2AsF6]: Static magnetic susceptibility
}

\author{
Mortensen, Kell; Tomkiewicz, Yaffa; Bechgaard, Klaus
}

Published in:

Physical Review B

Link to article, DOI:

10.1103/PhysRevB.25.3319

Publication date:

1982

Document Version

Publisher's PDF, also known as Version of record

Link back to DTU Orbit

Citation (APA):

Mortensen, K., Tomkiewicz, Y., \& Bechgaard, K. (1982). Antiferromagnetism in the organic conductor bis-

tetramethyltetraselenafulvalene hexafluoroarsenate [(TMTSF)2AsF6]: Static magnetic susceptibility. Physical Review B, 25(5), 3319-3325. https://doi.org/10.1103/PhysRevB.25.3319

\section{General rights}

Copyright and moral rights for the publications made accessible in the public portal are retained by the authors and/or other copyright owners and it is a condition of accessing publications that users recognise and abide by the legal requirements associated with these rights.

- Users may download and print one copy of any publication from the public portal for the purpose of private study or research.

- You may not further distribute the material or use it for any profit-making activity or commercial gain

- You may freely distribute the URL identifying the publication in the public portal 


\title{
Antiferromagnetism in the organic conductor bis-tetramethyltetraselenafulvalene hexafluoroarsenate [(TMTSF $\left.)_{2} \mathrm{AsF}_{6}\right]$ : Static magnetic susceptibility
}

\author{
Kell Mortensen \\ Physics Laboratory III, Technical University of Denmark, DK2800 Lyngby, Denmark* \\ and IBM T. J. Watson Research Center, Yorktown Heights, New York 10598 \\ Yaffa Tomkiewicz \\ IBM T. J. Watson Research Center, Yorktown Heights, New York 10598 \\ Klaus Bechgaard \\ Department of General and Organic Chemistry, H. C. Ørsted Institute, \\ University of Copenhagen, DK2100 Copenhagen, Denmark \\ (Received 8 September 1981)
}

\begin{abstract}
The anisotropy in the static magnetic susceptibility of bis-tetramethyltetraselenafulvalene hexafluoroarsenate [(TMTSF) ${ }_{2} \mathrm{AsF}_{6}$ ] has been investigated above and below the metal-to-insulator transition for a range of fields between 0.5 and $30 \mathrm{kG}$. The results are consistent with the expectations of a simple antiferromagnet with easy, intermediate, and hard axes close to the crystallographic $b^{*}, a$, and $c^{*}$ directions. Below the transition temperature $T_{c}=12 \mathrm{~K}$, a spin-flop transition is seen. The temperature dependence of the spin-flop critical field $H_{c}=4.5 \mathrm{kG}$ is insignificant. Above the transition temperature, evidence for magnetic crossover is observed. The experimental results show (TMTSF) ${ }_{2} X, X^{-}=\mathrm{AsF}_{6}{ }^{-}$and $\mathrm{PF}_{6}{ }^{-}$to be members of a family with quite similar physical properties. The data are discussed on the basis of Overhauser's treatment of itinerant antiferromagnetism.
\end{abstract}

\section{INTRODUCTION}

Since the class of organic conductors of the form (TMTSF) ${ }_{2} X$, based on the tetramethyltetraselenafulvalene (TMTSF) molecule was first synthesized in $1979,{ }^{1}$ it has attracted extensive interest. The compounds exhibit a variety of physical properties, which are different from those observed in other organic linear compounds. A series of the materials, where $X^{-}$is any of the symmetrical octahedral anions containing fluorine, $\mathrm{PF}_{6}{ }^{-}$, $\mathrm{AsF}_{6}{ }^{-}, \mathrm{SbF}_{6}{ }^{-}$, and $\mathrm{TaF}_{6}{ }^{-}$, has been reported to be superconducting at moderate hydrostatic pressure of $6.5 \mathrm{kbar}$ in the $(0.4-1.5)-\mathrm{K}$ region. ${ }^{2-5}$ At ambient pressure, these materials undergo a metal-toinsulator $(M I)$ transition close to $12 \mathrm{~K}^{1}$ Below the transition temperature, however, the dc conductivity remains relatively high. The experimental results for salts with tetrahedral anions containing oxygen are more divergent. While (TMTSF) $)_{2} \mathrm{ClO}_{4}$ remains metallic down to $1.3-1.5 \mathrm{~K}$, where a transition into a superconducting state occurs, ${ }^{6}$
(TMTSF) ${ }_{2} \mathrm{ReO}_{4}$ undergoes a $M I$ transition at 182 $\mathrm{K}$ (Ref. 7) and (TMTSF) ${ }_{2} \mathrm{IO}_{4}$ is insulating already at room temperature. ${ }^{8}$ Another tetrahedral $X^{-}$ salt (TMTSF) ${ }_{2} \mathrm{BF}_{4}$ is insulating below $39 \mathrm{~K}$.

The hexafluorophosphate salt has been most intensely examined. It was shown that magnetic and transport properties in the low-temperature insulating phase exhibit anomalous behavior. At the transition temperature $T_{c}$ the electron paramagnetic resonance (EPR) susceptibility was observed to disappear very sharply, ${ }^{9}$ whereas only minor effects were detected in static susceptibility measurements. ${ }^{1,10}$ Moreover, it was shown that both the dc conductivity and the spin paramagnetism are drastically affected by small electric fields for $T<T_{c} .{ }^{11,12}$ These observations led to the speculation that a spin-density wave might be responsible for the $M I$ transition. Subsequently, direct evidence for magnetic ordering was found in static susceptibility measurements. ${ }^{13}$ The static susceptibility showed an anisotropy and a spin-flop transition characteristic of an antiferromagnet having an 
easiest axis in the plane perpendicular to the stacking axis. Since the work on (TMTSF) ${ }_{2} \mathrm{PF}_{6}$, however, was done on an ensemble of crystals which were aligned only along the stacking axis $a$, these previous data did not uncover the orientation of the easy axis. ${ }^{13}$ (TMTSF) $)_{2} \mathrm{PF}_{6}$ was also studied by use of a nuclear magnetic resonance (NMR) technique. ${ }^{14}$ The results, which showed a vanishing ${ }^{77} \mathrm{Se}$ nuclear resonance signal and a broadening ${ }^{1} \mathrm{H}$ resonance, were attributed to the formation of spin-density waves.

In this paper we report static magnetic measurements of the same kind as those reported in Ref. 13 , but with much more experimental evidence. While the data on (TMTSF) ${ }_{2} \mathrm{PF}_{6}$ shown in Ref. 13 were performed on an ensemble of crystals aligned only along the stacking axis, the present work was done on one large single crystal. Hereby, the anisotropy effects could be studied in more detail, yielding quantitative results where only surmises were possible earlier. We report the first determination of the spin orientation below the $M I$ transition and a detailed analysis of the features of the spin-flop transition. The studies were performed on a single crystal of (TMTSF) ${ }_{2} \mathrm{AsF}_{6}$, the hexafluoroarsenate analog of $(\mathrm{TMTSF})_{2} \mathrm{PF}_{6}$. The physical properties of (TMTSF) ${ }_{2} \mathrm{AsF}_{6}$ appear to be very similar to those of the isomorphous $\mathrm{PF}_{6}^{-}$ salt. ${ }^{1,4,15}$ We observe magnetic behavior indicative of antiferromagnetic ordering occurring at $T_{c}=12$ $K$. At low magnetic fields an anisotropic spin susceptibility with three principal magnetic axes is seen for $T<T_{c}$. The easy axis, i.e., the orientation of the spins in the ordered state, is found to be close to the $b^{*}$ axis. Although the results perpendicular to the easy axis are less conclusive, our data suggest that the stacking axis $a$ is close to the intermediate axis and that the $c^{*}$ axis is close to the hard magnetic axis. Upon increasing the magnetic field parallel to the easy axis $\left(b^{*}\right)$, a spin-flop transition is observed at $H_{c}=4.5 \mathrm{kG}$. The antiferromagnetically-ordered spins then "flop" from the parallel to a transverse direction, most likely close to the $a$ axis.

\section{EXPERIMENTAL}

Single crystals of (TMTSF) ${ }_{2} \mathrm{AsF}_{6}$ were grown by an electrochemical oxidation of the neutral TMTSF molecule at constant current in $\mathrm{CH}_{2} \mathrm{Cl}_{2}$ solution containing the supporting electrolyte $n$ $\mathrm{Bu}_{4} \mathrm{NAsF}_{6}$. The resulting black needles were quite large, with masses up to $5 \mathrm{mg}$. From x-ray diffraction it is found that the needle axis is parallel to the stacking axis $(a)$ and that the largest face is a (001) plane. This is the same morphology as that found in (TMTSF) ${ }_{2} \mathrm{PF}_{6}{ }^{16}$

The static magnetic susceptibility was measured on a VTS susceptometer (SHE 801). The induced magnetic moment of the sample was determined by moving it between a pair of counterwound coils, each of which is connected to a superconducting quantum interference device (SQUID)-based fluxmeasuring system. The sample was glued to a cotton thread. The measurements were performed with the sample in three crystallographic orientations, with $a, b^{*}$, and $c^{*}$, respectively, parallel to the external magnetic fields $(\overrightarrow{\mathrm{H}}) . b^{*}$ and $c^{*}$ are perpendicular to the (010) face and the (001) face, respectively. With the angle between $b^{*}$ and $c^{*}$ equal to $84^{\circ}$, the $\left(a, b^{*}, c^{*}\right)$ basis is close to being orthogonal. We emphasize, though, that we have no proof that these directions coincide with the principal axes for the magnetic susceptibility tensor. However, as discussed below, the measurements show that the $a, b^{*}$, and $c^{*}$ axes are, at least, very close to the principal ones. The measured magnetic moments were corrected for contributions from glue and thread, which were measured separately. These contributions were, in all data obtained, less than $10 \%$ of the magnetic moment of the sample.

\section{RESULTS AND DISCUSSIONS}

In Figs. $1-3$ are shown the static magnetic susceptibilities, $\chi_{\text {total }}$ is the magnetization-field, versus temperature as measured with the external magnetic field $(\overrightarrow{\mathbf{H}})$ applied parallel to $a, b^{*}$, and $c^{*}$, respectively. The figures include data for different values of $\overrightarrow{\mathrm{H}}$. In all three configurations measured, we observe a significant temperature dependence below approximately $15 \mathrm{~K}$. In the same temperature region, $\chi_{\text {total }}$ measured with $\overrightarrow{\mathrm{H}}|| b^{*}, \chi_{b^{*}}$, exhibits strong field dependence, whereas those measured with $\overrightarrow{\mathrm{H}}|| a$ and $\overrightarrow{\mathrm{H}}|| c^{*}, \chi_{a}$ and $\chi_{c^{*}}$, respectively, remain almost unaffected. Between 15 and 30 $\mathrm{K}$, both the $T$ and the $\overrightarrow{\mathrm{H}}$ dependences are negligible. The constant values obtained in this temperature region are $-2.95,-2.55$, and $-2.75, \pm 0.05$ $\times 10^{-4} \mathrm{emu} / \mathrm{mole}$. The temperature dependences of $\chi_{a}$ and $\chi_{c^{*}}$ have several common properties. Close to $12 \mathrm{~K}$, they both show a characteristic anomaly. But while the $T$ dependence between 12 and $15 \mathrm{~K}$ of $\chi_{a}$ within the error bar is equal to that of $\chi_{b^{*}}$, the $T$ dependence of $\chi_{c^{*}}$ is much less pronounced. Well below $12 \mathrm{~K}, \chi_{a}$ and $\chi_{c^{*}}$ in- 


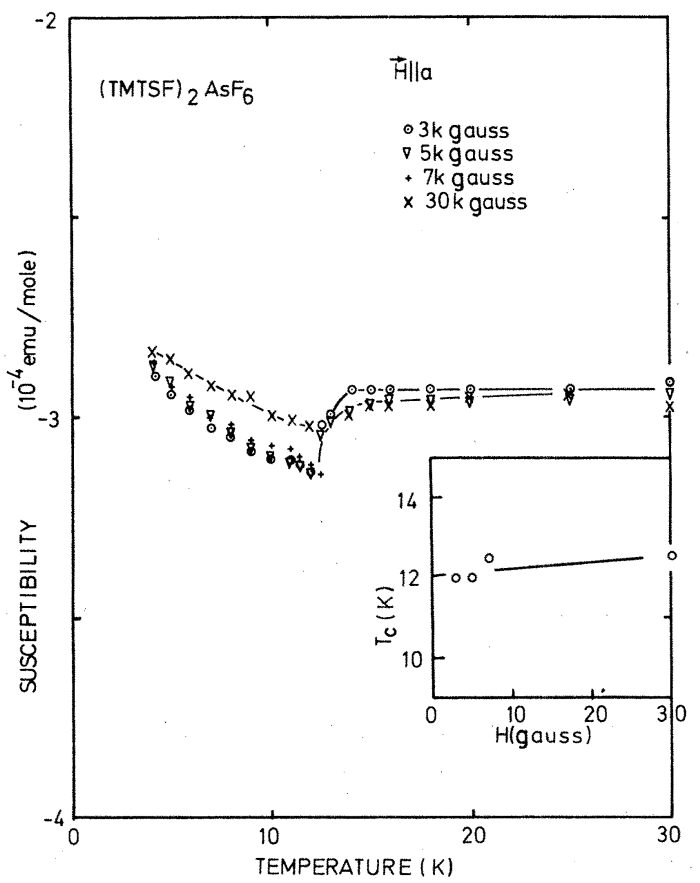

FIG. 1. Static magnetic susceptibility of

(TMTSF) ${ }_{2} \mathrm{AsF}_{6}$ vs temperature as measured with different fields parallel to $a$. The inset shows the transition temperature as a function of $\overrightarrow{\mathrm{H}} \| a$ as detected by the anomaly in $\chi$.

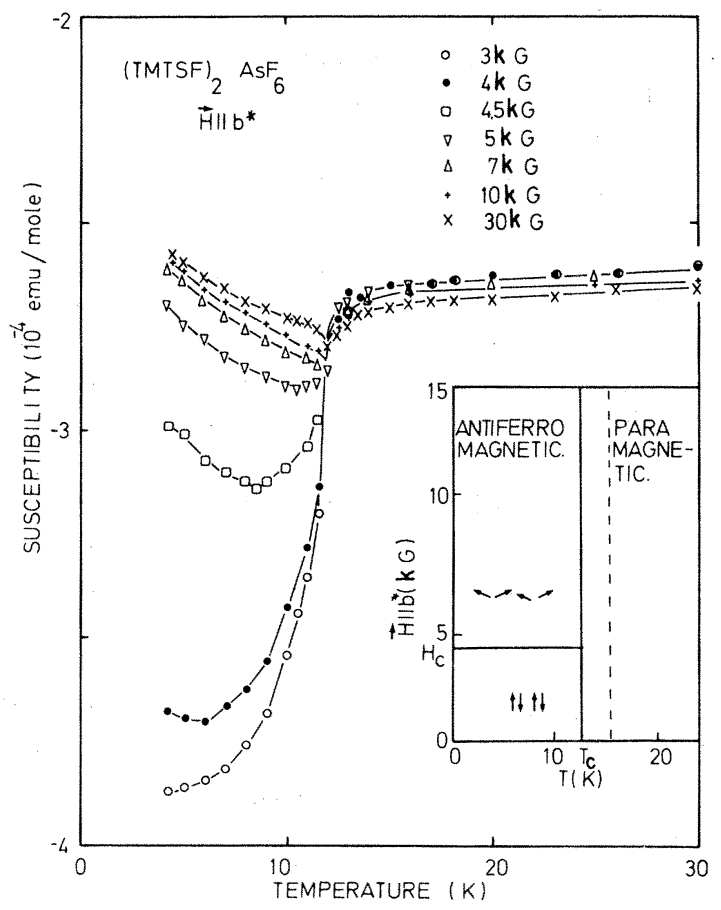

FIG. 2. Static magnetic susceptibility of $(\mathrm{TMTSF})_{2} \mathrm{AsF}_{6}$ vs temperature as measured with different fields parallel to $b^{*}$. The inset shows the phase diagram as determined from the $T$ and $\overrightarrow{\mathrm{H}}$ dependence of $\chi$.

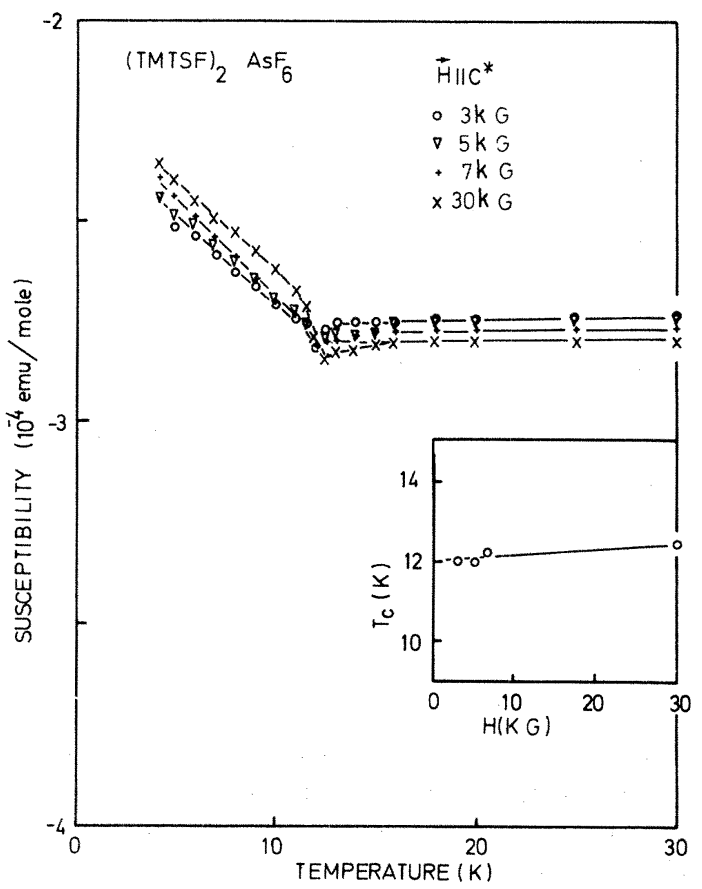

FIG. 3. Static magnetic susceptibility of $(\mathrm{TMTSF})_{2} \mathrm{AsF}_{6}$ vs temperature as measured with different fields parallel to $c^{*}$. The inset shows the transition temperature as a function of $\overrightarrow{\mathbf{H}} \| c^{*}$ as determined by the anomaly in $\chi$.

crease linearly with decreasing temperature, both with approximately the same slope. At high magnetic fields, the susceptibilities measured with $\overrightarrow{\mathrm{H}} \| a$ and $\overrightarrow{\mathbf{H}} \| b^{*}$ are indistinguishable.

In addition to the temperature dependence of the susceptibility, the characteristic field dependence is important in attempting to understand the lowtemperature ground state of (TMTSF $)_{2} \mathrm{AsF}_{6}$. As we have already mentioned, the results exhibit significant field dependence of the susceptibility for $\overrightarrow{\mathrm{H}}|| b^{*}$, and only minor, or none, for $\overrightarrow{\mathrm{H}} \| a$ and $\overrightarrow{\mathbf{H}}|| c^{*}$. These features are more clearly seen in Fig. 4 , which shows the susceptibilities (right scale on the figure) versus field as measured at a fixed temperature, $T=4.2 \mathrm{~K}$. Upon increasing magnetic field parallel to $b^{*}$, a rather sharp transition occurs at which the magnitude of the susceptibility changes. The critical field $H_{c} \simeq 4.5 \mathrm{kG}$ is seen from Figs. 3 and 5, to be roughly independent of temperature, for $T \leq T_{c}$. Well below and well above $\overrightarrow{\mathrm{H}}_{c}$, only minor field dependence of $\chi_{b^{*}}$ is seen. The jump in susceptibility,

$$
\Delta \chi=\chi_{b^{*}}\left(\overrightarrow{\mathrm{H}}>>\overrightarrow{\mathrm{H}}_{c}\right)-\chi_{b^{*}}\left(H<<H_{c}\right),
$$

varies as a function of temperature, as shown in 


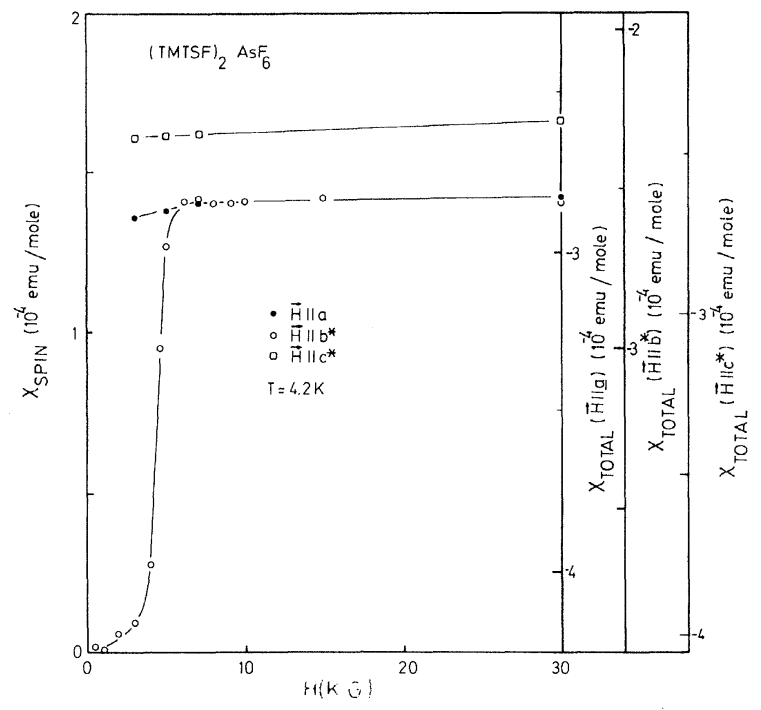

FIG. 4. Susceptibility vs fields, demonstrating spinflop when $\overrightarrow{\mathrm{H}}$ is applied parallel to the easy axis $\left(b^{*}\right)$. The right scales show the total susceptibilities, the left scale the spin susceptibility (see text).

the insert of Fig. 5. Close to $12 \mathrm{~K}, \Delta \chi$ decreases rapidly to zero.

The temperature and field dependences of the measured anisotropic susceptibility tensor are con-

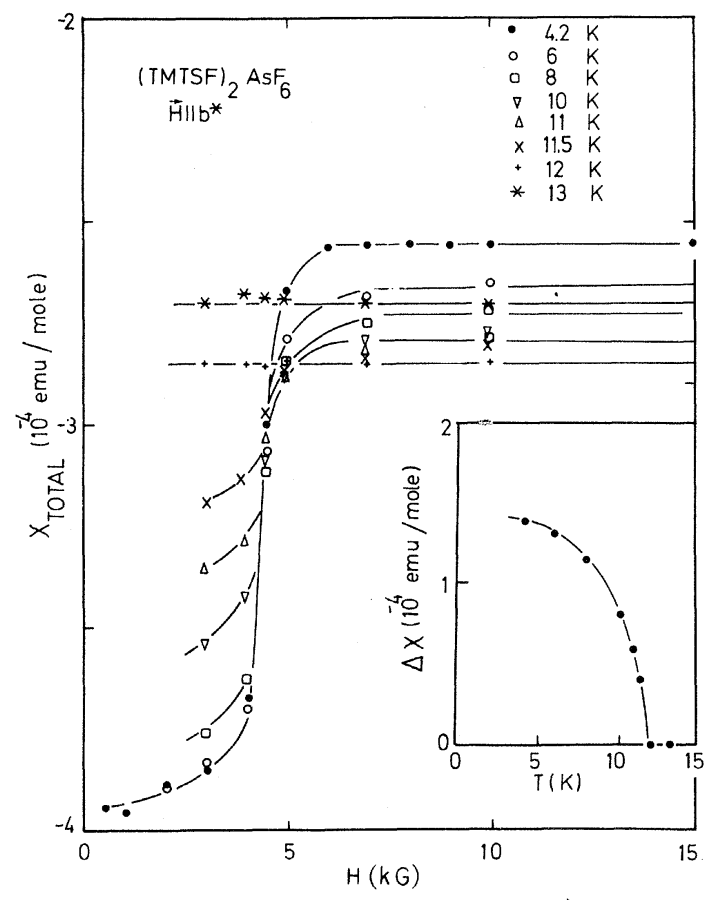

FIG. 5. Static magnetic susceptibility vs $\overrightarrow{\mathrm{H}} \| b^{*}$ as measured at different temperatures. The inset shows the drop $\Delta \chi$ in susceptibility versus temperature. sistent with expectations for a simple antiferromagnet characterized by having three principal magnetic axes. ${ }^{17}$ The easy axis $\sigma$ is close to the crystallographic $b^{*}$ axis. The intermediate and the hard magnetic axes are, most likely, close to $a$ and $c^{*}$, respectively. The susceptibilities measured with magnetic fields perpendicular to the easy axis, i.e., $\overrightarrow{\mathrm{H}} \| a$ and $\overrightarrow{\mathrm{H}} \| c^{*}$ in Figs. 1 and 3, are relatively large because the sublattices in these configurations are optimally suited to being canted by a weak field. Both $\chi_{a}$ and $\chi_{c^{*}}$ have extrapolated zerotemperature values which are slightly larger than the saturated values in the paramagnetic state close to $T_{c}$. That may be because in the ordered state the field can interact optimally with all spins, in contrast to the paramagnetic state. Other physical effects may be important, too. An indication of these is the fact that the spin susceptibility should be isotropic within the plane perpendicular to the spin orientations. In contrast to the results of fields perpendicular to $b^{*}$, the spin susceptibilities of $\overrightarrow{\mathrm{H}}$ parallel to the easy axis are small, since any finite spin moment can only occur as a result of some spins turning over, which is more or less prevented by the exchange field.

The distinct field dependence of $\chi_{b^{*}}$ and the absence of any field dependence of $\chi_{a}$ and $\chi_{c^{*}}$ shows that (TMTSF) ${ }_{2} \mathrm{AsF}_{6}$ undergoes a spin-flop transition at $\overrightarrow{\mathrm{H}}_{c} \cdot{ }^{17}$ When the external magnetic field exceeds the critical value, the antiferromagnetically ordered spins turn from the direction of the easy axis into a predominantly perpendicular one (see inset of Fig. 2). This produces the sharp increase in the susceptibility, as is seen in Fig. 5. If the external field were applied exactly along the easy axis $\overrightarrow{\mathbf{H}} \| \sigma$, the spin-flop would occur via a firstorder transition, thus producing a discontinuous jump in $\chi_{\sigma}$. For slight misorientation, however, the susceptibility increases continuously. ${ }^{18}$ The rather abrupt spin-flopping experimentally obtained, therefore, indicates that any deviation between the easy axis and the $b^{*}$ axis must be relatively small.

The temperature dependence of the discontinuity in susceptibility $\Delta \chi$ is shown in the insert of Fig. 5. Down to $6 \mathrm{~K}$, we find that $\Delta \chi$ scales with the relative temperature $T-T_{c}$ like

$$
\Delta \chi \propto\left(T-T_{c}\right)^{\alpha},
$$

as has been predicted for an uniaxial antiferromagnet. ${ }^{19}$ The exponent $\alpha$ is determined by the specific heat and anisotropic crossover. For $(\mathrm{TMTSF})_{2} \mathrm{AsF}_{6}$, we find $\alpha \simeq 0.46$. More detailed 
studies, which include that of the BCS-like gap behavior, must still be done in order to compare this value with the one obtained from scaling theory.

The interpretations given above are based on the assumption that the molecular diamagnetism is relatively temperature and field independent. A1though it is known that $\chi_{\mathrm{dia}}$ of one compound HMTSF-TCNQ actually does exhibit some irregular $T$ dependences, ${ }^{20}$ we believe that in (TMTSF) ${ }_{2} \mathrm{AsF}_{6}$ this is a reasonable assumption. The interpretations are, moreover, based on the concept of sublattice magnetization. Although such a theory is more obvious for localized spins, it is also appropriate for itinerant spins, which we believe these to be. A phenomenological theory may be based on Overhauser's Hartree-Fock treatment. ${ }^{21}$ The antiferromagnetic spin-density wave (SDW) ground state is in this model favored by almost planar Fermi surface ${ }^{22}$ characteristic of this class of highly anisotropic materials. The Overhauser mechanism can also account for the semiconducting transport behavior below $T_{c},{ }^{1}$ because in a quasi-one-dimensional system the SDW gap covers the whole Fermi surface.

The origin of the magnetic anisotropy may be due to magnetic dipole-dipole interactions or spinorbit coupling. An estimate of the dipolar term is particularly sensitive to the Se-Se distances, since the spin densities essentially are concentrated on the selenium atoms. ${ }^{23}$ The difference between the shortest intrastack and interstack Se-Se contacts, 3.778 and $3.905 \AA$, respectively, ${ }^{15}$ is only $0.031 \AA$ in (TMTSF) ${ }_{2} \mathrm{AsF}_{6}$. Moreover, the four selenium atoms in TMTSF have different nearest-neighbor contacts. Detailed calculations are therefore necessary in order to understand the magnetic anisotropy experimentally obtained.

The spin susceptibility $\chi_{s}(\overrightarrow{\mathbf{H}}|| \sigma)$ in an Overhauser antiferromagnet is depressed by the gap energy $\Delta$. Near zero temperature, $\chi_{s}(\overrightarrow{\mathbf{H}} \| \sigma)$ vanishes as $\exp (-\Delta / k T)$. As the temperature approaches $T_{c}$, the susceptibility rises quite sharply since the gap is diminishing rapidly. The EPR absorption signal in the antiferromagnetic state is shifted compared to that in the paramagnetic state, since it becomes an antiferromagnetic resonance (AFMR). This behavior is consistent with the experimental observations of $\chi_{b^{*}}$ measured at low magnetic fields, and preliminary AFMR studies. ${ }^{24}$ The Overhauser gap $\Delta$ can thus be obtained from the $\chi_{b^{*}}(T)$ behavior. The results $\Delta / k \simeq 30 \mathrm{~K}$ are slightly larger than the value obtained from trans- port data. ${ }^{1}$

According to the arguments given above, the susceptibility remaining when we extrapolate $\chi_{b^{*}}(T)$ to zero temperature is the contribution due to molecular diamagnetism, i.e.,

$\chi_{\text {dia }}\left(\overrightarrow{\mathrm{H}} \| b^{*}\right)=(-3.95 \pm 0.05) \times 10^{-4} \mathrm{emu} / \mathrm{mole}$.

This number should be compared with the value estimated from Pascal's constants, $\chi_{\text {dia }}^{p}=-4.00$ $\times 10^{-4} \mathrm{emu} /$ mole. $^{25}$ By using the measured value of the diamagnetism for HMTSF and adjusting for the lack of methylene linkage and the Pascal contribution of $\mathrm{AsF}_{6}$ in the present compound we obtain a molecular diamagnetism of

$\chi_{\mathrm{dia}}^{p^{\prime}}=-3.54 \times 10^{-4} \mathrm{emu} / \mathrm{mole}^{10,20}$ Both estimates give values which are in reasonable agreement with the experimentally obtained susceptibility.

More insight into the molecular diamagnetism may be gained by referring to data obtained by the EPR technique. ${ }^{26}$ These studies suggest that the high-temperature $(T \gtrsim 15 \mathrm{~K})$ spin susceptibility is isotropic. If we now assume that $\chi_{\text {dia }}$ is relatively temperature independent, i.e., $\chi_{\text {dia }}(20 \mathrm{~K}) \simeq \chi_{\text {dia }}(0)$, the parameters along $a$ and $c^{*}$ can be evaluated too. The results are

$$
\chi_{\mathrm{dia}}(\overrightarrow{\mathbf{H}} \| a) \simeq(-4.25 \pm 0.05) \times 10^{-4} \mathrm{emu} / \mathrm{mole}
$$

and

$$
\chi_{\mathrm{dia}}\left(\overrightarrow{\mathbf{H}}|| c^{*}\right) \simeq(-4.05 \pm 0.05) \times 10^{-4} \mathrm{emu} / \mathrm{mole},
$$

respectively.

In Fig. 4 (left scale) and Fig. 5 are shown the spin susceptibilities as deduced by subtracting the diamagnetic corrections given above, for $H=3 \mathrm{kG}$ and $H=7 \mathrm{kG}$. The low-field data $\left(H<<H_{c}\right)$ in Fig. 4 clearly show the anisotropic feature below $T_{c}$, with three different magnetic axes. The highfield data $\left(H>>H_{c}\right)$ in Fig. 4 show that the spin susceptibility measured with $\overrightarrow{\mathrm{H}}|| b^{*}$ is equal to that with $\overrightarrow{\mathrm{H}} \| a$, thus suggesting that the $c^{*}$ axis is magnetically harder than $a$. Above $T_{c}$, in Figs. 6, and 7 we also observe a region of a few degrees with some anisotropic properties, indicative of magnetic precursor developing below approximately $15 \mathrm{~K}$. While the spin susceptibility of $\overrightarrow{\mathrm{H}}|| a$ and $\overrightarrow{\mathrm{H}}|| b^{*}$ in this temperature region are equal within the error bars, both showing a rapid fall in $\chi_{\text {spin just above }}$ $T_{c}$, the value of $\overrightarrow{\mathrm{H}} \| c^{*}$ is distinctly different. $\chi_{\text {spin }}\left(\overrightarrow{\mathbf{H}}|| c^{*}\right)$ exhibits only a small temperature dependence between 12 and $15 \mathrm{~K}$. The precursor may reflect a crossover in spin behavior. The an- 


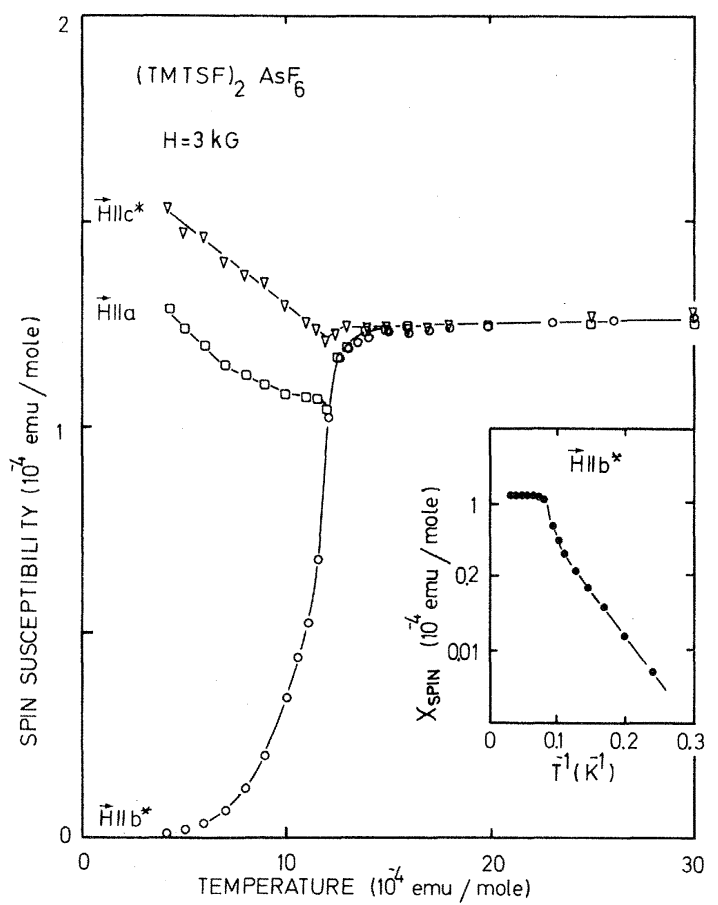

FIG. 6. Spin susceptibility of (TMTSF) ${ }_{2} \mathrm{AsF}_{6}$ as deduced from static measurements with field parallel to $a$, $b^{*}$, and $c^{*}$, respectively (see text). The magnetic field $H=3 \mathrm{kG}$ is less than the critical spin-flop field.

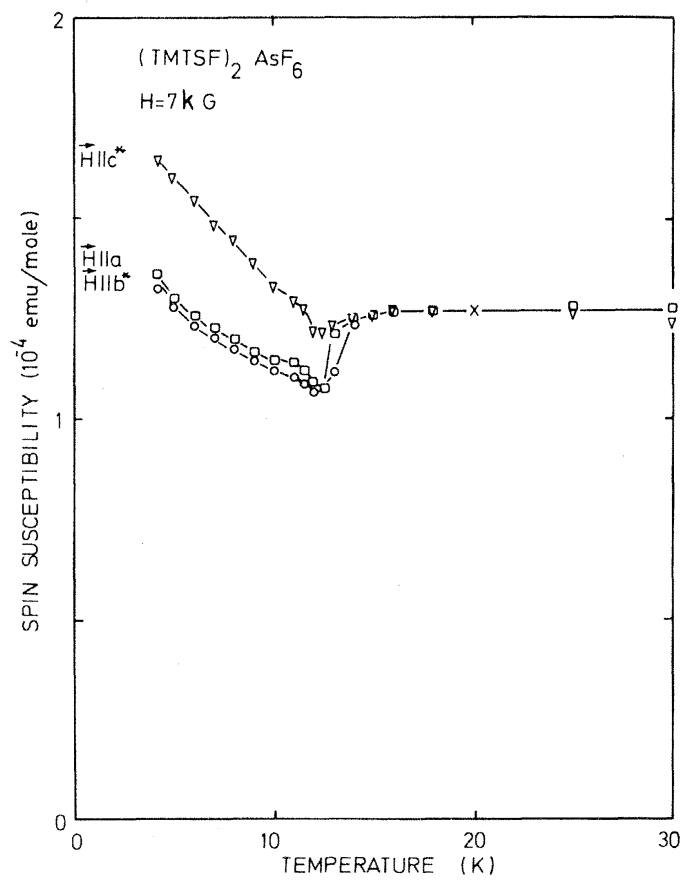

FIG. 7. Spin susceptibility of (TMTSF) ${ }_{2} \mathrm{AsF}_{6}$ as deduced from static measurements with field parallel to $a$, $b^{*}$, and $c^{*}$, respectively (see text). The magnetic field $H=7 \mathrm{kG}$ is larger than the critical spin-flop field. isotropy of the spin susceptibility suggests then that the spin motion in the crossover region is dominated by easy plane geometry rather than the easy axis symmetry, developed below $T_{c}$.

These results lead to the phase diagram schematically shown in the insert of Fig. 2. The roughly field-independent value of $T_{c}$, and the temperature-independent value of $\overrightarrow{\mathrm{H}}_{c}$ are obtained from the data shown in Figs. 2 and 5, respectively. The $T$ dependences of the magnetic anomalies shown in Figs. 1 and 3 suggest, though, that the transition temperature is slightly sensitive to the magnitude of $\overrightarrow{\mathrm{H}} \perp b^{*}$. Like the behavior of (TMTSF) ${ }_{2} \mathrm{PF}_{6},{ }^{13}$ these results show a small increase in $T_{c}$ upon increasing magnetic field.

The magnetic data of $(\mathrm{TMTSF})_{2} \mathrm{AsF}_{6}$ presented above support the data and interpretations previously given for (TMTSF) ${ }_{2} \mathrm{PF}_{6} \cdot{ }^{13}$ In fact, the results suggest that the spin behavior of these two isostructural compounds are not only qualitatively the same, but also quantitatively. ${ }^{27}$ For example, if we take the low-field data measured with $\overrightarrow{\mathrm{H}}$ parallel to the stacking axis, ${ }^{13}$ the spin susceptibilities agree within a few percent in the whole temperature region studied, $4.2-30 \mathrm{~K}$. Likewise, the spin susceptibility of (TMTSF) ${ }_{2} \mathrm{PF}_{6}$ as measured with the magnetic field perpendicular to the stacking axis of an assembly of crystals with the $a$ axes aligned, but $b^{*}$ and $c^{*}$ pointing randomly perpendicular to $a,{ }^{13}$ agrees within a few percent with the average of the spin susceptibilties of (TMTSF) ${ }_{2} \mathrm{AsF}_{6}$ measured with $\overrightarrow{\mathrm{H}} \| b^{*}$ and $\overrightarrow{\mathrm{H}}|| c^{*}$.

The diamagnetic correction of (TMTSF) ${ }_{2} \mathrm{PF}_{6}$ in these comparisons is found by arbitrarily, but certainly successfully, setting the values of $\chi_{s}$ equal at $20 \mathrm{~K}$. The diamagnetic corrections of (TMTSF) ${ }_{2} \mathrm{PF}_{6}$ is hereby found to be $\chi_{\text {dia }}$ $=-3.5 \pm 0.3 \mathrm{emu} / \mathrm{mole}$.

\section{SUMMARY}

(TMTSF $)_{2} \mathrm{AsF}_{6}$ has been found to have an antiferromagnetic ground state. The antiferromagnetic transition, which occurs at $12 \mathrm{~K}$, can properly be understood as a result of the Overhauser mechanism. The antiferromagnetic ground state is characterized by having three principal magnetic axes; the easy, the intermediate, and the hard axes are close to $b^{*}, a$, and $c^{*}$ respectively. Below the transition temperature, a spin-flop transition is clearly 
seen at $4.5 \mathrm{kG}$. The spin orientation then flops from being parallel to $b^{*}$ to an orientation predominantly parallel to $a$. Between approximately 15 and $12 \mathrm{~K}$, there may be a magnetic crossover, dominated by easy plane symmetry. It is found that the magnetic properties of (TMTSF) ${ }_{2} X$, $X^{-}={P F_{6}}^{-}$, and $X^{-}=\mathrm{AsF}_{6}^{-}$are very similar, indicating that the antiferromagnetic behavior discussed here is not unique to one compound.

\section{ACKNOWLEDGMENTS}

We are pleased to acknowledge illuminating discussions with Dr. T. D. Schults, Dr. J. B. Torrance, Jr., and Dr. H. J. Pedersen. We also thank Dr. N. Thorup for his help in determining the crystal morphology. One of us (K.M.) has been supported by IBM World Trade and the Danish Natural Science Research Council (11-2216).
${ }^{*}$ Permanent address.

${ }^{1} \mathrm{~K}$. Bechgaard, C. S. Jacobsen, K. Mortensen, H. J. Pedersen, and N. Thorup, Solid State Commun. $\underline{33}$, 1119 (1980).

${ }^{2}$ D. Jérome, A. Mazaud, M. Ribault, and K. Bechgaard, J. Phys. (Paris) Lett. 41, L95 (1980).

${ }^{3}$ K. Andres, F. Wudl, D. B. McWhan, G. A. Thomas, D. Nalewajek, and A. L. Stevens, Phys. Rev. Lett. 45, 1449 (1980).

${ }^{4}$ R. Ribault, J. P. Pouget, D. Jérome, and K. Bechgaard, J. Phys. (Paris) Lett. 41, L607 (1980).

${ }^{5}$ S. S. P. Parkin, M. Ribault, D. Jérome, and K. Bechgaard, (unpublished).

${ }^{6} \mathrm{~K}$. Bechgaard, K. Carneiro, M. Olsen, F. B. Rasmussen, and C. S. Jacobsen, Phys. Rev. Lett. $\underline{46}$, 852 (1981).

${ }^{7}$ C. S. Jacobsen, H. J. Pedersen, K. Mortensen, G. Rindorf, N. Thorup, J. B. Torrance, and K. Bechgaard (unpublished).

${ }^{8}$ E. M. Engler, Y. Tomkiewicz, K. Mortensen, and R. L. Greene, Bull. Am. Phys. Soc. 26, 214, (1981).

${ }^{9}$ H. J. Pedersen, J. C. Scott, and K. Bechgaard, Solid State Commun. 35, 207 (1980).

10J. C. Scott, H. J. Pedersen, and K. Bechgaard, Phys. Rev. Lett. 45, 2125 (1980).

${ }^{11}$ W. M. Walsh, F. Wudl, G. A. Thomas, D. Nalewajek, J. J. Hauser, and P. A. Lee, Phys. Rev. Lett. 45 , 829 (1980).

12P. M. Chaikin, G. Grüner, E. M. Engler, and R. L. Greene, Phys. Rev. Lett. 45, 1874 (1980).

${ }^{13}$ K. Mortensen, Y. Tomkiewicz, T. D. Schultz, and E. M. Engler, Phys. Rev. Lett. 뜨, 1234 (1981).

${ }^{14}$ A. Andrieux, D. Jérome, and K. Bechgaard, J. Phys.
(Paris) Lett. 42, L90 (1981).

${ }^{15} \mathrm{~F}$. Wudl, (unpublished).

${ }^{16} \mathrm{~N}$. Thorup, private communication.

${ }^{17}$ See, for example, S. V. Vonsovskii, Magnetism (Wiley, New York, 1974).

${ }^{18}$ I. S. Jacobs, J. Appl. Phys. 2ㅡ, 61S (1961).

${ }^{19}$ M. E. Fisher, and D. R. Nelsen, Phys. Rev. Lett. 2, 1350 (1974).

${ }^{20} \mathrm{G}$. Soda, D. Jérome, M. Weger, K. Bechgaard, and E. Pedersen, Solid State Commun. 20, 107 (1076).

${ }^{21}$ A. W. Overhauser, Phys. Rev. 128, 1437 (1962).

22P. A. Fedders and P. C. Martin, Phys. Rev. 143, 245 (1966).

${ }^{23}$ R. M. Metzger, J. Chem. Phys. 75, 482 (1981).

${ }^{24}$ J. B. Torrance, Jr., H. J. Pedersen, and K. Bechgaard, private communication.

${ }^{25}$ Pascal's constants used were taken from E. König [Magnetic Properties of Coordination and Organometallic Transition Metal Compounds, Landolt-Börnstein Group II, edited by K.-H. Hellwege (Springer, Berlin, 1966), Vol. 2.] H, 2.93; C 6.00; Se, 23; P, 26.3; As, 20.9; F, 6.3; constitutive corrections $\mathrm{C}=\mathrm{C},-5.5$ (all in units of $-1 \times 10^{-6} \mathrm{emu} / \mathrm{mole}$ ).

${ }^{26}$ H. J. Pedersen, J. C. Scott, and K. Bechgaard (unpublished).

${ }^{27}$ Our conclusion that the spin susceptibility of (TMTSF) ${ }_{2} \mathrm{PF}_{6}$ and (TMTSF) $)_{2} \mathrm{AsF}_{6}$ at $T \gtrsim T_{c}$ are equal within our experimental error, both being $(1.35 \pm 0.1) \times 10^{-4} \mathrm{emu} / \mathrm{mole}$, is inconsistent with the results obtained by EPR technique, Refs. 9 and 26. While the EPR results of the $\mathrm{PF}_{6}$ salt agree with our value, the EPR susceptibility of the $\mathrm{AsF}_{6}$ salt is much larger. 